\title{
Serological screening for sexually transmitted infections in pregnancy: is there any value in re-screening for HIV and syphilis at the time of delivery?
}

\author{
D C Qolohle, A A Hoosen, J Moodley, A N Smith, K P Mlisana
}

\begin{abstract}
Objective-The aim of this study was to assess the prevalence of syphilis, human immunodeficiency virus (HIV), and hepatitis $B$ virus (HBV) infections in women at the time of delivery, and to determine the seroconversion rates for syphilis and HIV infections from initial booking visit to delivery.

Setting-The labour ward of a typical tertiary hospital in a developing country and serving an indigent African population.

Method-Four hundred and eighteen women presenting in labour were randomly selected and informed consent obtained for serological testing for syphilis and HBV infections in umbilical cord blood samples. The specimens were then given a study number, the gestational ages recorded and anonymously tested for HIV infection.

Results-Of the 191 women who had antenatal care, $13(6 \cdot 8 \%)$ were HIV antibody positive at the initial "booking" visit. An additional 4 were found to be HIV antibody positive at the time of delivery resulting in a seroconversion rate of $2 \cdot 2 \%$. The seroconversion rate for syphilis at the time of delivery was $2 \cdot 7 \%$. Hepatitis $B$ surface antigens were detected in only 2 women, one of whom was antigen positive.

Conclusion-The high seroconversion rates for both syphilis and HIV infection in pregnancy justifies re-screening for these conditions in endemic areas such as ours.
\end{abstract}

(Genitourin Med 1995;71:65-67)

Keywords: screening, pregnancy, syphilis, HIV

\section{Introduction}

The practice of screening for medical disorders and infectious diseases at the first antenatal visit is well established. At King Edward VIII Hospital (KEH), Durban, a large referral centre serving mainly an indigent population, serological screening for syphilis and human immunodeficiency virus (HIV) infection is performed at the initial antenatal visit. It has been recommended, however, that in high prevalence areas, screening for syphilis be repeated in late pregnancy and at the time of delivery to reduce the incidence of congenital syphilis.

Opai-Tetteh et al, working at $\mathrm{KEH}$, have reported a seroconversion rate of $3 \%$ for syphilis at the time of delivery. ${ }^{2}$ This finding is highly relevant for a condition which is eminently detectable and curable. Furthermore, Mlisana et al have shown that the "unbooked mother" has a much higher prevalence of syphilis than regular antenatal attenders. ${ }^{3}$ The prevalence of HIV infection in women attending antenatal care in the Natal-Kwa Zulu has been shown to be steadily increasing and was approximately $5 \%$ for $1992 .{ }^{4}$ The prevalence of HIV infection, however, at the time of delivery is not known.

As far as we are aware, screening for hepatitis $B$ virus (HBV) infection in pregnancy is not routinely performed in South Africa. ${ }^{56}$ Hepatitis B virus screening in pregnancy has advantages; vertical transmission is a possibility in mothers who are hepatitis $\mathrm{B}\left(\mathrm{HB}_{\mathrm{e}}\right)$ antigen positive. Consequently, screening may identify such mothers for further investigation, and prevalence rates in antenatal populations may help to prioritise groups for $\mathrm{HBV}$ vaccination.

The aim of this study, therefore, was to assess the prevalence of syphilis, HIV and HBV infections in umbilical cord blood samples at the time of delivery, and to evaluate the seroconversion rate of syphilis and HIV infections from initial booking visit to delivery.

\section{Patients and methods}

The study was conducted in the labour ward, $\mathrm{KEH}$ over a 2 month period in May and June 1993. Four hundred and eighteen women were randomly selected and informed consent obtained for serological testing for syphilis and HBV infections in umbilical cord blood. The samples were then allocated a study number, the gestational ages recorded and anonymously tested for HIV infection by categorising them into three groups: Group A consisted of booked women who had at least two antenatal visits and records of serological screening for syphilis and HIV; Group B consisted of women who were referred following booking visits at community clinics and regional hospitals; Group C consisted of unbooked women who had no antenatal care.

Human immunodeficiency virus antibody testing was performed using a recombinant $\mathrm{HIV}_{1} / \mathrm{HIV}_{2}$ Elisa kit (Abbott Laboratories, Wiesbaden, Germany) for screening at first antenatal visit and delivery. All positive sera were confirmed by Western blot (Diagnostic biotechnology, Singapore). Hepatitis B sur- 
face antigen $\left(\mathrm{HB}_{\mathrm{s}} \mathrm{Ag}\right)$, e antigen and core IgM antibody were tested for by radioimmunoassay (Abbott Laboratories, North Chicago, USA). The rapid plasma reagin (RPR) test (RPR Becton Dickenson BBL Microbiology, Maryland, USA) was used to screen for syphilis and positive sera confirmed by Treponema pallidum haemaglutination (TPHA) test (Fujirebio, Tokyo, Japan) or the fluorescent treponemal antibody absorption (FTA-ABS) test (Behring Diagnostics, Marburg, Germany). Serological testing for antenatal screening has been centralised and performed at one laboratory serving the greater Durban area. Hence the same tests (serological kits) are used for all specimens.

Statistics

Prevalence of HIV and syphilis in the three groups were compared using McNemar's chi square test. Fishers exact test was used for pairwise comparisons where expected cell sizes were less than 5 . A $p$ value $\leqslant 0.05$ was regarded as statistically significant.

\section{Results}

Laboratory results for HIV antibody, syphilis serology and $\mathrm{HB}_{\mathrm{s}}$ antigen at the time of delivery for the 418 women are shown in table 1 . One hundred and ninety one $(45 \cdot 7 \%)$ women were booked, Group A; $172(41 \cdot 2 \%)$ were referred, Group B; and $55(13.2 \%)$ were unbooked, Group C. Overall, 32 (7.7\%) women of the study population were HIVpositive at the time of delivery (95\% CI: $5 \cdot 1-10 \cdot 3)$. Seventeen $(8 \cdot 9 \%)$ women in group A were HIV positive (95\% CI: $4 \cdot 8-13 \cdot 0)$. This group consisted of 13 women who were HIV positive at the initial booking visit and an additional four who seroconverted during the prenatal period, giving a seroconversion rate of $2 \cdot 2 \%$, that is, 4 of 178 (95\% CI: $0 \cdot 0-4 \cdot 4)$. These women had booked at 17, 23, 24 and 28 weeks gestation and all delivered at term.

The HIV seroprevalence for groups B and C were $4 \cdot 1 \%(95 \% \mathrm{CI}: 1 \cdot 1-7 \cdot 1)$ and $14 \cdot 6 \%$ (95\% CI: $5 \cdot 2-23 \cdot 9)$ respectively. This was a

Table 1 Laboratory results of all serological tests at delivery

\begin{tabular}{llll}
\hline Group & $\begin{array}{l}\text { HIV antibody } \\
\text { No. positive (\%) }\end{array}$ & $\begin{array}{l}\text { Syphilis } \\
\text { No. positive (\%) }\end{array}$ & $\begin{array}{l}\text { HBs } A g \\
\text { No. positive (\%) }\end{array}$ \\
\hline A (n=191) & $17(8 \cdot 9)$ & $16(8 \cdot 4)$ & $1(0 \cdot 5)$ \\
B (n=172) & $7(4 \cdot 1)$ & $13(7 \cdot 6)$ & $1(0 \cdot 6)$ \\
C (n=55) & $8(14 \cdot 6)$ & $10(18 \cdot 2)$ & $0(0 \cdot 0)$ \\
Total 418 & $32(7 \cdot 7)$ & $39(9 \cdot 3)$ & $2(0 \cdot 5)$ \\
\hline
\end{tabular}

HIV = Human immunodeficiency virus

$\mathrm{HB}_{\mathrm{s}} \mathrm{Ag}=$ Hepatitis $\mathrm{B}$ surface antigen

Table 2 Results of serological tests for syphilis

\begin{tabular}{|c|c|c|c|}
\hline \multirow[b]{2}{*}{ Group } & \multirow{2}{*}{$\begin{array}{l}\text { At first visit } \\
R P R \text { positive } \\
T P H A+/ F T A A B S+\end{array}$} & \multicolumn{2}{|l|}{ At delivery } \\
\hline & & $\begin{array}{l}R P R \text { positive } \\
T P H A+\mid F T A A B S+\end{array}$ & $\begin{array}{l}R P R \text { negative } \\
T P H A+/ F T A A B S+\end{array}$ \\
\hline $\begin{array}{l}\text { A }(n=191) \\
B(n=172) \\
C(n=55) \\
\text { Total }\end{array}$ & $\begin{array}{l}18(9 \cdot 4) \\
16(9 \cdot 3) \\
34(9 \cdot 4)\end{array}$ & $\begin{array}{l}16(8 \cdot 4) \\
13(7 \cdot 6) \\
10(18 \cdot 2) \\
39(9 \cdot 3)\end{array}$ & $\begin{array}{l}17 \\
22 \\
11 \\
50(12 \cdot 0)\end{array}$ \\
\hline
\end{tabular}

RPR = Rapid Plasma Reagin test

TPHA = Treponema pallidum Haemaglutination Test

FTA-ABS = Fluorescent Treponemal Antibody Absorption Test

FTA-ABS $=$ in brackets
. Flu significant difference $(p=0.011)$. However, there were no statistically significant differences between groups $A$ and $C(8.9 \%$ vs $14 \cdot 6 \%)$ or groups A and B $(8 \cdot 9 \%$ vs $4 \cdot 1 \%)$. Positive syphilis serology at delivery ranged from $7 \cdot 6 \%$ to $18 \cdot 2 \%$ for the different groups. Hepatitis B surface antigens were detected in only two women, one booked and one referred. One of these was also e antigen positive. None of the other samples demonstrated evidence of $\mathrm{HB}_{\mathrm{s}} \mathrm{Ag}$, e antigen and core IgM antibody.

Results for syphilis serology at initial visit and at delivery are shown in table 2 . Thirty nine of $418(9 \cdot 3 \%)$ women of the study population had reactive syphilis serology at delivery (95\% CI: $6 \cdot 5-12 \cdot 1)$, whilst 34 of $363(9 \cdot 4 \%)$ tested positive for syphilis at the first antenatal visit. Nine of 329 women who had negative serological tests for syphilis at initial booking were found to have reactive syphilis serology at delivery, giving a seroconversion rate of $2 \cdot 7 \%(95 \%$ CI: $0 \cdot 1-4 \cdot 5)$. The time interval for seroconversion in these 9 women ranged from 2 to 15 weeks. Eighteen (5.0\%) women with reactive sera at booking became nonreactive at delivery. The period from booking to delivery ranged from 4 to 17 weeks for this group.

Syphilis sero-prevalence at delivery was $8.4 \%$ for Group A (95\% CI: $4 \cdot 5-12 \cdot 3) ; 7.6 \%$ for group B (95\% CI: $3 \cdot 6-11 \cdot 6)$ and $18 \cdot 2 \%$ for group C (95\% CI: 4.1-28.4). There was a significant difference between groups $A$ and $C$ $(p=0.037)$ as well as groups B and C $(p=$ $0.023)$. However, there was no significant difference in the prevalence between groups $A$ and $B(8.4 \%$ vs $7 \cdot 6 \%)$. A further fifty $(12.0 \%)$ women had evidence of previous exposure to syphilis as judged from the fact that their RPR tests were non-reactive, whereas their confirmatory tests (TPHA or FTA-ABS) were positive.

\section{Discussion}

Recently Opai-Tetteh $e t a l^{2}$ and Delport $e t a l^{7}$ have recommended rescreening for syphilis at the time of delivery in an attempt to decrease the morbidity and mortality associated with congenital syphilis. This suggestion stems from the belief that congenital syphilis can be prevented by effective maternal screening and treatment during the antenatal period. Our finding of a significantly higher prevalence of syphilis in "unbooked" women than women who have had antenatal care $(18.2 \%$ vs $8.4 \%$ ), a high level of past exposure to syphilis (RPR-negative and confirmatory tests positive $12.0 \%$ ), as well as a seroconversion rate of $3 \%$ in women who have had antenatal care confirm the findings of Opai-Tetteh et $a l^{2}$ and Mlisana et al. ${ }^{3}$ Furthermore these findings demonstrate that syphilis is endemic in the Natal-KwaZulu region.

Although Bam et $a l^{8}$ found positive syphilis serology in $15 \%$ of pregnant women at the time of first testing and only $8 \%$ in cord blood, our experience has shown a good correlation between maternal sera and cord blood testing. ${ }^{23}$ 
Rescreening in pregnancy for infectious diseases should be the ideal in areas of high prevalence. In the Natal-KwaZulu region prevalence figures for HIV infection amongst antenatal attenders have been increasing in a stepwise manner from $1.6 \%$ in $1990,2.9 \%$ in 1991 and $4 \cdot 8 \%$ in $1992 .{ }^{4}$ Our finding of $7 \cdot 7 \%$ at the time of delivery confirms this increasing trend. More importantly, the prevalence was higher in "unbooked" pregnant women $(14.6 \%)$. Furthermore the seroconversion rate for HIV-negative women from the time of initial testing to delivery was $2 \cdot 2 \%$. To our knowledge such a finding has not been reported at any other centre. These findings are indicative of endemic HIV and argue strongly for rescreening for both HIV and syphilis at the time of delivery in areas such as ours.

It is of interest that there was a higher prevalence of HIV antibody in women who booked at KEH than those referred from community clinics and district hospitals $(8.9 \%$ vs $4.1 \%)$. However, this was not the case for women who had positive syphilis serology. We cannot explain this difference and further analyses may be of value.

A number of studies have shown that the route of transmission for $\mathrm{HBV}$ infection in the neonate in Africa is horizontal rather than vertical. ${ }^{6}$ In contrast, HBV neonatal infection in South-East Asia is predominantly vertical. Our findings in cord blood of $0.5 \%$ ( 2 of 418 women) surface antigen positivity not only confirms this low prevalence amongst pregnant African women but also suggests that the mode of HBV transmission locally is usually horizontal.
In conclusion, this study like previous reports from $\mathrm{KEH}$ has established that NatalKwaZulu is an area of high prevalence for both syphilis and HIV infection. It is well recognised that screening for HIV infection during pregnancy is of value because it enables appropriate counselling, early termination of pregnancy if indicated, advice on breast feeding, education on the value of protective intercourse and follow-up of the infant. Consequently in areas of high seroprevalence of both syphilis and HIV disease our findings justify rescreening for both these conditions at the time of delivery. On these grounds we strongly recommend re-screening for both syphilis and HIV disease in pregnancy.

We thank Sandy Moodley for her patience in preparation of the manuscript and the MRC of South Africa for its support.

1 Centers for Disease Control. Sexually transmitted diseases treatment guidelines. $M M W R$ 1989;38:9-10.

2 Opai-Tetteh ET, Moodley J, Hoosen AA. Re-screening for syphilis at the time of delivery in an area of high prevalence. $S$ Afr Med F 1993;83:725-6.

3 Mlisana KP, Monokoane S, Hoosen AA, Moodley J Adhikari P, Taylor L. Syphilis in the "unbooked" preg nant women. $S$ Afr Med $\mathscr{F} 1992 ; 82: 18-20$.

4 Report of Department of National Health. South African Third National HIV survey of women attending antenatal clinics, South Africa. Epidemiological Comment 1993;20:35-47.

5 Guidozzi F, Song E, Scoub BD, Johnson S. Should pregnant urban, South African women be screened fo hepatitis B? S Afr Med F 1992;83:103-5.

6 Botha JF, Ritchie MJJ, Dusheiko GM, Menton HWK, Kew MC. Hepatitis B virus carrier state in black children in Ovamboland: Role of perinatal and horizontal infection. Lancet 1984;2:1210-2.

7 Delport SD, Ballard RC, Cameron NA, Rothberg AD Prevention of congenital syphilis by effective maternal Prevention of congenital syphilis by effective maternal 83:710-1.

8 Bam RH, Cronje HS, Muir A, Griessel DJ, Hoek BB. Syphilis in pregnant patients and their offspring. Int $\mathscr{f}$ Gynecol Obstet 1994;44:113-8. 